\title{
Role of Canagliflozin on function of CD34+ve endothelial progenitor cells (EPC) in patients with type 2 diabetes
}

Seshagiri Rao Nandula2,3, Nabanita Kundu ${ }^{2}$, Hassan B. Awal ${ }^{1}$, Beda Brichacek ${ }^{2}$, Mona Fakhri ${ }^{2}$ Nikhila Aimalla ${ }^{2}$, Adrian Elzarki ${ }^{1,2}$, Richard L. Amdur ${ }^{1}$ and Sabyasachi Sen ${ }^{1,2,3^{*}}$ (D)

\begin{abstract}
Background: Endothelial progenitor cells (EPCs) has been shown to be dysfunctional in both type 2 diabetes mellitus (T2DM) and chronic kidney disease (CKD) leading to poor regeneration of endothelium and renal perfusion. EPCs have been shown to be a robust cardiovascular disease (CVD) risk indicator. Effect of sodium glucose channel inhibitors (SGLT2i) such as Canagliflozin (CG) on a cellular biomarker such as CD34+ve progenitor cells, which may help predict CVD risk, in patients with T2DM with established CKD has not been explored.

Methods: This is a pilot study where 29 subjects taking metformin and/or Insulin were enrolled in a 16 week, double blind, randomized placebo matched trial, with a low dose $100 \mathrm{mg} \mathrm{CG}$ as the intervention group compared to matched placebo. Type 2 diabetes subjects (30-70 years old), with hemoglobin A1c ( $\mathrm{HbA1c}$ ) of 7-10\%, were enrolled. CD34+ve cell number, migratory function, gene expression along with vascular parameters such as arterial stiffness, serum biochemistry pertaining to cardio-metabolic health, resting energy expenditure and body composition were measured. Data were collected at week 0, 8 and 16. A mixed model regression analysis was done and $p$ value less than 0.05 was considered statistically significant.
\end{abstract}

Results: A significant expression of CXCR4 receptor with a concomittant increase in migratory function of CD34+ve cells was observed in CG treated group as compared to placebo group. Gene expression analysis of CD34+ve cells showed an increase in expression of antioxidants (superoxide dismutase 2 or SOD2, Catalase and Glutathione Peroxidase or GPX) and notable endothelial markers (PECAM1, VEGF-A, and NOS3). A significant reduction in glucose and $\mathrm{HbA1c}$ levels were observed along with improved systolic and diastolic blood pressure in the CG group. A significant increase in adiponectin $(p=0.006)$ was also noted in treatment group. Urinary exosomal protein leak in urine, examining podocyte health (podocalyxin, Wilm's tumor and nephrin) showed reduction with CG

Conclusion: Low dose Canagliflozin has a beneficial effect on CD34+ cell function, serum biochemistry and urinary podocyte specific exosomes in type 2 diabetes.

Keywords: Canagliflozin, Type 2 diabetes, Progenitor cells, Endothelial function, Urinary exosomes

*Correspondence: ssen1@gwu.edu

2 Department of Medicine, The George Washington University, 2300 Eye Street, SMHS, Room 462, Washington, DC 20037, USA

Full list of author information is available at the end of the article

\section{Background}

Diabetes mellitus is a major public health concern worldwide as number of patients with type 2 diabetes mellitus (T2DM) is increasing rapidly and it is gradually reaching the proportions of a world epidemic. Currently, 463 million adults are living with diabetes and by 
2045 estimated number of adult living with diabetes will reach to 700 million $[1,2]$. Centers for Disease Control and Prevention (CDC) reported approximately, 9.4\% of American adults have diabetes [1,2]. Diabetes is closely associated with oxidative stress, inflammation, and as a result endothelial dysfunction and cardiovascular complications [1-3]. On the other hand, $15 \%$ of US adults are estimated to have chronic kidney disease and $38 \%$ of end stage renal disease developed Chronic kidney disease (CKD) as a consequence of diabetes [4]. Use of a sodiumglucose linked transporter inhibitor (SGLT-2i) has shown promise in improving glycemic control, weight reduction, hypertension and even changes in circulating reninangiotensin-aldosterone system (RAAS) and nitric oxide (NO) $[5,6]$. However, whether these group of drugs have any effect on cardiovascular disease (CVD) risk modification or specifically on endothelial cell or endothelial progenitor cells, is unclear. It is extremely important to evaluate improvement, especially cardiovascular and endothelial health of the patient after administration of diabetic drugs.

Available therapeutics are focused on glycemic control to delay the diabetes related complications [7, 8]. Widely used oral drugs to control hyperglycemia in patient with diabetes are metformin, dipeptidyl peptidase 4 (DPP4) inhibitors, and SGLT2 inhibitors. Several studies showed SGLT2 inhibitor can be beneficial compared to DPP4 inhibitors, especially on subjects with type 2 diabetes and mild chronic kidney disease. A use of a SGLT-2 inhibitor has a promise to improve glycemic control, obesity and hypertension as reported by several research groups [913]. Meta analysis studies have shown less risk of heart failure with SGLT2 inhibitors in comparison with DPP4 inhibitor and Glucagon-like peptide-1 (GLP1) agonist [14]. However, recently several studies have shown canagliflozin (CG) specifically lower the risk of cardiovascular disease in pre-clinical and clinical studies in type 2 diabetes [15-21]. Recent studies suggested CG may inhibit endothelial pro-inflammatory chemokine/cytokine secretion through $5^{\prime}$ adenosine monophosphate-activated protein kinase (AMPK) dependent and independent mechanisms [22] and CG even helps to improve myocardial ischemia-reperfusion injury [23].

Serum based biomarkers are being used for long time to identify and monitor cardiovascular improvement. However, use of serum based inflammatory markers are inefficient and does not change until the endothelium is already damaged or inflamed and produce paracrine pro-inflammatory molecules which subsequently gets detected [24]. For this reason serum based biomarkers are unable to appropriately detect effect on endothelium at an early stage. Several studies recently has shown that endothelial progenitor cells (defined as CD34+ve cell) can serve as an important biomarker to monitor cardiovascular function [25-29].

Endothelial progenitor cells (EPC) defined as CD34+ve progenitor cells are obtained from the hematopoietic stem cell pool by magnetic bead column separation (MACS Miltenyi Biotech). EPCs contribute to vascular repair, angiogenesis and also vasculogenesis [28-31].

It is well established that EPCs respond to a chemoattractant such as stromal derived factor 1 alpha (SDF1 $\alpha)$. The EPCs migrate towards a chemoattractant such as SDF1a, released by wounded/damaged/ apoptosis prone tissue $[30,31]$ This process of targetted migration of EPCs has been described as "homing-in" [26]. The recruitment of EPCs help to repair the damaged tissue. It is also known that EPCs release or secrete growth factors and cytokines that promote regeneration as part of their paracrine properties [32, 33]. We reported earlier that CD34+ve cells help to monitor effect of a therapy or intervention in an early onset $[26,28,29]$. A few preclinical and clinical studies showed, improvement of cardiovascular health by using a combination of DPP-4 inhibitor and metformin in type 2 diabetic patient by improving endothelial dysfunction and vascular complications from diabetes by increasing number of CD34+ve EPCs and migratory capacity $[29,34]$. There are also some previous studies that indicate that SGLT2 inhibitors may help to improve endothelial dysfunction both in patients and animal studies [35-40], however such studies don't give us much information at a cellular level.

Previous studies from our laboratory have shown that CD34+ve cells, derived from peripheral blood can act as a cellular biomarker that is more reliable than serum based markers for CVD risk estimation [26, 28, 29]. Arterial stiffness assays also help define endothelial function independent of serum assays but corelates well to standard biochemical assays that are used to define endothelial function [41, 42]. In our previous studies using two di-peptylpeptidase inhibitors (DPP4i) such as saxagliptin and linagliptin in type 2 diabetes mellitus (T2DM) patients $[43,44]$ we have demonstrated that CD34+ve cells are responsive to a change in therapy or intervention within 2-4 weeks using CD34+ as a biomarker and CD34+ cell based assays can be used as a reliable non serum based cellular bio-marker. The same is true for of a prediabetes population with an aerobic exercise intervention, where changes in gene expression are quite fast [29]. CD34+ve cells or endothelial progenitor cells have been used clinically to improve collateral circulation and have been extensively studied as a robust cardiovascular biomarker. Therefore, studying CD34+ve cells in T2DM subjects with or without Canagliflozin can give vital information about the medication and its effect on hematopoetic stem cell pool that may have a long-term 
effect on future endothelium and other vascular cells. Our non cellular outcome measures were focused on parameters that are agreed to be indicators of endothelial function such as arterial stiffness, serum inflammatory markers and markers of renal podocyte health.

In this 16-week intervention study we investigated the synergistic effect of canagliflozin, a SGLT2 inhibitor and metformin, on number and function of CD34+ve EPCs while adjusting for variables that may affect stem cell number and function such as hemoglobin A1c (HbA1C), body mass index (BMI), gender and age.

\section{Methods}

\section{Trial design and oversight}

This is a phase 4 (post-marketing), two arm, single site, parallel group, double blind, placebo controlled randomized clinical trial comparing low dose Canagliflozin $100 \mathrm{mg}$ tablets, taken orally, once daily, with matching placebo. The study was conducted in accordance with Good Clinical Practice guidelines set forth by the International Conference of harmonization and any local regulatory guidelines with the approval and oversight of the George Washington University Institutional Review Board. The trial was funded by Janssen Scientific Affairs, LLC and conducted by the Investigator-Sponsor Dr. Sabyasachi Sen at the George Washington University.

Subjects were initially pre-screened to assess eligibility. Once determined preliminary eligibility, they were brought in for a screening visit to confirm eligibility via interview, medical record check and laboratory workup once the subject signed the informed consent. The subjects $(n=29)$ were then enrolled into one of two arms of the study: $100 \mathrm{mg}$ Canagliflozin or matching placebo. 15 subjects were enrolled into the active canagliflozin group and 14 subjects were enrolled into the placebo group.

There were 3 study visits total, first at week 0 , second at week 8 (mid-point) and third and last visit at week 16 . All three of the visits had same assessments. The assessments that were done were: vital measurements, adverse event (AE) check and a peripheral blood draw. Approximately $80 \mathrm{ml}$ of blood was drawn for CD34+ve endothelial progenitor cell harvesting and routine blood work.

Other parameters tested were resting metabolic rate (RMR, energy expenditure), measurement of waist to hip ratio, urine sample collection, Tanita body composition scale, pulse wave analysis and pulse wave velocity to determine arterial stiffness. Subjects were advised to adhere to $150 \mathrm{~min}$ of weekly aerobic exercise and their activity levels were monitored using ACTi graph activity monitor.

A follow up phone call visit was done 30 days from the last in-person visit to assess for any residual adverse events (AE).

\section{Participants}

Subjects were included if they were between 30 and 70 years old inclusive, with a diagnosis of Type 2 Diabetes for 15 years or less. HbA1c inclusions were between 7.0 and $10.0 \%$ inclusive. Their baseline medications were stable dose of Insulin (either short acting or long acting) and/or Metformin (1-2 g/day). A stable dose was considered to be at least the maximum labeled dose or dose not associated with unacceptable side effects. Patients with BMI between 25 and $39.9 \mathrm{~kg} / \mathrm{m}^{2}$ were included, thereby excluding severe obesity. Only, patients with impaired renal function were included, with Chronic Kidney Disease stage 1 to 3, with lowest eGFR cut-off of $30 \mathrm{ml} / \mathrm{min} / 1.73$ (GFR, as calculated by MDRD formula). During the duration of the study alterations in baseline medications were done to keep HbA1C between 7 and $8 \%$ across all subjects.

Any patients with Type I diabetes, history of Diabetic ketoacidosis, low hematocrit (less than 28 units), history of recent pancreatitis or cancer, recent coronary or cerebrovascular event within 6 months, use of consistent steroid medications, untreated thyroid disease were excluded.

Additional Inclusion and Exclusion criteria can be found in Appendix.

\section{Outcome objectives}

The primary objective is to ascertain if addition of Canagliflozin improves CD34+ve cell number, (CD34+ve number, \%CD34+ve of total Mononuclear Cell population) function (cell migration function in response to SDF $1 \alpha$ ) and gene expression, in Type 2 Diabetes Mellitus, which will be correlated to improvement in $24 \mathrm{~h}$ urinary protein estimation and serum Creatinine Clearance.

The secondary objective is to correlate the cellular outcome measures with other measures of endothelial function such as Arterial Stiffness [measured by pulse wave analysis (Augmentation Index) and pulse wave velocity $(\mathrm{m} / \mathrm{s})$ ], Serum Biochemistry (CMP, IL6, hsCRP, Leptin, Serum insulin, TNF $\alpha$ ), Adiposity (as \% body fat), resting energy expenditure (in kcal) and Glycemic control (through HbA1c).

\section{Body composition measurement}

Body composition was measuring using Tanita ${ }^{\mathrm{TM}}$ BF-350 Body Composition Scale and manually. Manual measurement included height, waist circumference, hip circumference. Tanita scale (Tanita Corporation of America, Inc, USA) uses a bio-impedance electrical impulse to measure body fat percent, fat mass $(\mathrm{kg})$, fat free mass $(\mathrm{kg})$, percent 
body water, water mass $(\mathrm{kg})$ alongside weight. It then calculates the BMI and estimated basal metabolic rate.

\section{Basal metabolic rate measurement}

Resting Emergency Expenditure (REE) was measured using KORR REEVUE (Korr Medical Technologies, USA). Test was conducted with the subject sitting and well rested. Subject was instructed to keep a tight seal around the mouthpiece and use the nose clip to avoid breathing in from the nose. The test ran for about $10 \mathrm{~min}$. It calculated estimated REE, predicted REE, estimated Total Energy Expenditure (TEE), $\mathrm{VO}_{2}$ Max and estimated calorie intake per day.

\section{Arterial stiffness}

This parameter was measured using Atcor Sphygmocor CP system (Atcor Technologies, USA). We obtained two outcomes such as: pulse wave velocity and pulse wave analysis. The patient was supine on the examination table, three leads were attached on right forearm, left forearm and left shin.

Pulse wave analysis (PWA) was measured on the left Radial Artery with the subject supine. At least three readings were taken with Operator Index $\geq 80$. Measurement includes Augmentation Index (AI), Augmentation Index adjusted for Heart Rate of 75 (AI-75), Augmentation Pressure (AP), Aortic and Radial reading of systolic, diastolic, pulse pressure and mean pressure.

Pulse wave velocity (PWV) was measured with the subject supine. This measurement requires a distal and proximal artery. Distal was used as right femoral artery with proximal being the left carotid. Index and ring fingers were used to manually localize the pulse, sometimes an arterial Doppler was used to localize the femoral pulse on patient with challenging body habitus. Once a stable pulse waveform was observed, the probe position was kept stable for 20 more pulses before the reading was finalized. Three readings were taken with standard deviation of less than $10 \%$. The result reported a velocity in $\mathrm{m} / \mathrm{s}$, alongside the standard deviation with error.

\section{Biological sample and vital collection}

A venous blood sample was collected from the Antecubital fossa. About $80 \mathrm{ml}$ of blood was collected. $60 \mathrm{ml}$ for EPC analysis and $20 \mathrm{ml}$ for standard of care blood works which included Basic Metabolic Panel, Lipid Panel, HbA1c, hsCRP, IL6, Adiponectin and Insulin. Enzyme linked immune assay (ELISA) was performed to analyze serum GLP1 and SDF1 $\alpha$ using ELISA Immunoassay kit (Raybiotech, Norcross, GA) for GLP1 and Sandwich ELISA (EHCXCL12A, Thermo Scientific, USA) for SDF1 $\alpha$. Urine sample was collected for urine Microalbumin and Creatinine ratio. Vitals were gathered on the left arm, Systolic Pressure, Diastolic Pressure and heart Rate, along with sublingual temperature. Serum nicotinamide adenine dinucleotide (NAD/NADH) were measured by using NAD/NADH assay kit from Abcam, MA, USA (Catalog No. ab65348) and Ketone bodies were from serum were measured by using Ketone Body Assay Kit from Millipore Sigma, MO, USA (Catalog No. MAK134).

\section{ACTi graph activity monitor}

Subjects level of activity was measured using Actigraph wGT3x-BT activity monitors (ActiGraph Inc, USA). Subjects were advised on diet and exercise instructed to wear the meter during all waking hours and was advised to adhere to $150 \mathrm{~min}$ of moderate intensity aerobic exercise per week. Actigraph served as a measure of this exercise compliance, and to verify for exercise as a confounding variable.

\section{Polyethylene glycol (PEG) enrichment of extracellular vesicles}

The cells debris and large apoptotic bodies were removed from the urine samples by centrifugation at $500 \times g$ for $5 \mathrm{~min}$ followed by $3000 \times g$ for $30 \mathrm{~min}$ at $4{ }^{\circ} \mathrm{C}$. Transfer supernatant into ultracentrifugation tubes and centrifuged at $100,000 \times g$ at $4{ }^{\circ} \mathrm{C}$ for $75 \mathrm{~min}$ (Optimal XPN-100 centrifuge, Beckmann Coulter Inc, USA). After ultracentrifugation the pellet was dissolved in RIPA buffer with protease inhibitor cocktail and stored the sample at $-80^{\circ} \mathrm{C}$ for further analysis.

\section{Extracellular vesicle characterization and Western blotting}

Extracellular vesicles were isolated by ultracentrifugation and identified by the expression of CD9, CD81, CD63 AND HSP70 markers in western blot. Extracellular vesicle extracts were fractionated by SDS-PAGE and transferred to a polyvinylidene difluoride membrane using a transfer apparatus according to the manufacturer's protocols (Bio-Rad, USA). After incubation with $5 \%$ nonfat milk in TBST (Bio-Rad, USA) (10 mM Tris, pH 8.0, $150 \mathrm{mM} \mathrm{NaCl}, 0.5 \%$ Tween 20) for $60 \mathrm{~min}$. The membrane was washed once with TBST and incubated with antibodies against CD9 (1:1000), CD81 (1:1000), CD63 (1:1000), HSP70 (1:1000) (Sysetem Biosciences, USA), anti-podocalyxin (PODXL, 1:1000), anti-wilms tumor protein $(1: 1000)$ and anti-Nephrin antibody $(1: 1000)$ at $4{ }^{\circ} \mathrm{C}$ for $12 \mathrm{~h}$ (Abcam, USA). Membranes were washed three times for $10 \mathrm{~min}$ and incubated with a 1:20,000 dilution of horseradish peroxidase-conjugated goat antirabbit antibody for $90 \mathrm{~min}$ at room temperature. Blots were washed with TBST three times and developed with Pierce ECL kit (ThemoFisher Scientific, USA). 
Cellular and clinical assessments CD34+ve endothelial progenitor cell analysis

Peripheral blood samples (approximately $60 \mathrm{ml}$ ) were drawn from patients and phosphate buffered saline (1:1) was added. Identification and quantification of circulating cell phenotypes was performed on fresh blood samples, within $3 \mathrm{~h}$ after collection, using flow cytometry. Briefly, mononuclear cells (MNCs) were then isolated from whole blood using a Ficoll density centrifuge method. MNCs were counted and aliquot was used for CFU-Hill colony formation assay following the manufacturer's instruction (Stem Cell Technologies, Vancouver, $\mathrm{BC}$, Canada). Colony forming unit was counted at day14. A fraction of the MNC were stained with fluorescein isothiocyanate (FITC)-conjugated antihuman CD34, Allophycocyanin (APC) conjugated antihuman CD184 (CXCR4) and FITC conjugated antihumanCD31 antibodies (Miltenyi Biotec $\mathrm{GmbH}$, Bergisch-Gladback, Germany) in order to analyze specific progenitor cell surface marker (CD34) and mature endothelial cell surface markers (CD31) or receptor for SDF1a ligand, CXCR4) by flow cytometry. After gating mononuclear cells in the side scatter (SSC)-A vs forward scatter (FSC)-A plot, CD34/CD184 single- and double-positive cells were identified. Cells were acquired on a fluorescence-activated cell sorter (FACS) Canto instrument (Becton Dickinson, USA) and scored with the FloJo software.

To isolate EPCs (CD34+ve), MNCs were magnetically sorted through a column after cells were stained with $\mathrm{CD} 34+$ ve microbeads antibody (Miltenyi Biotec $\mathrm{GmbH}$, Bergisch Gladback, Germany). An aliquot of CD34+ve cells were then stained with trypan blue and counted using an Auto Cellometer Mini (Nexcelom Bioscience, USA) to assess viability.

CD34+ve gene expression analysis was performed by quantitative reverse transcriptase polymerase chain reaction (qRT-PCR) as previously described [33]. CD34+ve cell total mRNA was extracted and purified using the RNeasy Minikit (Qiagen, Germany). mRNA was then converted into cDNA by using the high capacity cDNA reverse transcriptase kit (Thermo Fisher Scientific, MA) Possible gene expression changes promoted by Canagliflozin was assessed by a CFX96 real-time PCR systems (Bio-Rad, CA) using Taqman Universal masters Mix II (Thermo Fisher Scientific, USA) and inventoried probes. The gene expression analysis included antioxidants, apoptosis, endothelial functions, chemotaxis, inflammation and endothelial lineage cell surface markers. The expression of each individual gene was normalized to either housekeeping $18 \mathrm{~S}$ or GAPDH and calculated using $\mathrm{C}$-ddct method considering the difference in cycle threshold between visit 2 and 3 and baseline (visit 1).
The migratory capacity of CD34+ve was evaluated using the CytoSelect 24-well Cell Migration Assay kit (Cell Biolads, Inc., San Diego, CA). Cells were suspended in Serum free media and seeded at 100,000 cells per insert. Migration of the cells through a $3 \mu \mathrm{m}$ polycarbonate membrane to the wells containing a serum-free media (control) and chemoattractant SDF-1 $\alpha$ (10 or $100 \mathrm{ng} /$ ml) (from Sigma-Aldrich, USA) was assessed after cells were kept overnight in incubator. Migratory cells were dissociated from the membrane and subsequently lysed and quantified by fluorescence (480 nm/530 nm) using CyQuant GR dye (Cells Biolabs, Inc, USA). The fluorescence ratios between cells exposed to the chemotactic factor and cells exposed to chemoattractant-free media (control) along the visits were used to analyze the migratory capacity of the cells.

\section{Statistical analysis}

Continuous variable distributions were examined for skewness or outliers using histograms. When these were present, we log-transformed the variables. Outliers $>5 \mathrm{SD}$ from the mean were capped at 5SD from the mean. We used 2-tailed between-groups t-tests to examine differences between treatment groups at baseline on continuous variables, and either chi-square or the Fishers Exact test for categorical variables. To examine differences between treatment groups across all time points, as well as time effects, and whether the slope of change over time differed between treatments, we used random effects mixed model regression, examining the main effects of treatment (Canagliflozin vs. placebo), and time (v1, v2, v3), and the treatment by time interaction. This method allows us to use all non-missing subject data and adjusts for within-subject auto-correlation. For variables with significant effects in the mixed models, we examined the means graphically. SAS (version 9.4, Cary, NC) was used for data analysis with $\mathrm{p}<0.05$ considered significant.

Since subjects were randomized to treatment, chance of baseline subject characterizations acting as confounders are minimized. Therefore, randomized control trials do not usually adjust for baseline differences. However, in small studies, imbalances may exist as a result of random group assignment, and may thus function as mediators of an association between treatment and outcome. In this situation, one would need to adjust for the mediators in order to obtain the direct effect of treatment on outcome, which is the effect of interest.

\section{Results}

\section{Primary outcome}

The study population was representative of subjects with uncontrolled type 2 diabetes, but with no preexisting macro-vascular complications. All adverse effects 
Table 1 Blood biochemistry before and after Canagliflozin treatment

\begin{tabular}{|c|c|c|c|c|c|c|c|}
\hline \multirow[t]{2}{*}{ Parameter } & \multicolumn{3}{|c|}{ Placebo $(n=14)$} & \multicolumn{3}{|c|}{ Canagliflozin $(n=15)$} & \multirow{2}{*}{$\begin{array}{l}\text { Fixed } \\
\text { effects } \\
\text { test } \\
\text { P value }\end{array}$} \\
\hline & Visit 1 & Visit 2 & Visit 3 & Visit 1 & Visit 2 & Visit 3 & \\
\hline eGFR & $85.22 \pm 4.43$ & $79.85 \pm 4.22$ & $88.33 \pm 4.52$ & $80.24 \pm 4.27$ & $81.13 \pm 4.56$ & $79.90 \pm 4.21$ & 0.31 \\
\hline Creatinine & $0.84 \pm 0.05$ & $0.94 \pm 0.05$ & $0.80 \pm 0.06$ & $0.96 \pm 0.05$ & $0.88 \pm 0.06$ & $0.95 \pm 0.05$ & 0.37 \\
\hline $\mathrm{HDL}$ & $51.56 \pm 3.60$ & $52.23 \pm 3.66$ & $51.67 \pm 3.69$ & $42.96 \pm 3.47$ & $46.97 \pm 3.48$ & $46.17 \pm 3.48$ & 0.28 \\
\hline Cholesterol & $163.40 \pm 10.09$ & $166.23 \pm 10.53$ & $147.76 \pm 10.61$ & $167.78 \pm 9.74$ & $176.26 \pm 9.84$ & $171.57 \pm 9.84$ & 0.20 \\
\hline LDL/HDL & $1.68 \pm 0.15$ & $2.26 \pm 0.15$ & $1.60 \pm 0.16$ & $2.19 \pm 0.15$ & $1.43 \pm 0.16$ & $2.08 \pm 0.15$ & 0.92 \\
\hline C-reactive protein & $1.03 \pm 0.2$ & $0.40 \pm 0.2$ & $0.92 \pm 0.2$ & $0.54 \pm 0.2$ & $0.96 \pm 0.2$ & $0.63 \pm 0.2$ & 0.44 \\
\hline Insulin & $2.49 \pm 0.21$ & $2.63 \pm 0.20$ & $2.71 \pm 0.22$ & $2.50 \pm 0.20$ & $2.46 \pm 0.22$ & $2.56 \pm 0.20$ & 0.42 \\
\hline Augmentation Index (Al) & $22.14 \pm 2.9$ & $24.58 \pm 3.1$ & $21.37 \pm 3.2$ & $24.2 \pm 2.8$ & $23.21 \pm 2.9$ & $28.67 \pm 2.8$ & 0.17 \\
\hline PWV & $11.19 \pm 0.64$ & $10.85 \pm 0.68$ & $11.42 \pm 0.70$ & $9.59 \pm 0.66$ & $9.70 \pm 0.65$ & $10.12 \pm 0.63$ & 0.86 \\
\hline Leptin & $39.84 \pm 3.34$ & $42.42 \pm 3.52$ & $40.28 \pm 3.65$ & $28.36 \pm 3.30$ & $29.92 \pm 3.36$ & $27.79 \pm 3.29$ & 0.96 \\
\hline SDF-1a & $6.34 \pm 0.47$ & $5.74 \pm 0.45$ & $5.85 \pm 0.47$ & $5.49 \pm 0.46$ & $6.41 \pm 0.48$ & $5.68 \pm 0.44$ & 0.51 \\
\hline 3Hydroxy-butyric acid & $0.03 \pm 0.01$ & 0.0 & $0.03 \pm 0.01$ & $0.01 \pm 0.01$ & $0.001 \pm 0.01$ & $0.02 \pm 0.01$ & 0.75 \\
\hline Acetoacetic acid & $0.72 \pm 0.25$ & 0.0 & $0.30 \pm 0.27$ & $1.08 \pm 0.24$ & $0.05 \pm 0.25$ & $0.69 \pm 0.24$ & 0.86 \\
\hline
\end{tabular}

that occurred throughout the duration of the study were either not related to the study medication and design or fell within the expected side effects profile for Canagliflozin. Table 1 shows blood biochemistry measures across the three visits. No statistical significance was observed between the groups for body composition measures. Mean percent body fat is low and mean percent body water is high in canagliflozin group and showed no significance difference throughout the study (Table 2). This implies that the changes that we note in cellular and

Table 2 Cellular parameters before and after Canagliflozin treatment

\begin{tabular}{|c|c|c|c|c|c|c|c|}
\hline \multirow[t]{2}{*}{ Parameter } & \multicolumn{3}{|l|}{ Placebo $(n=14)$} & \multicolumn{3}{|c|}{ Canagliflozin $(n=15)$} & \multirow{2}{*}{$\begin{array}{l}\text { Fixed } \\
\text { effects } \\
\text { test } \\
\text { P value }\end{array}$} \\
\hline & Visit 1 & Visit 2 & Visit 3 & Visit 1 & Visit 2 & Visit 3 & \\
\hline Mean MNC & $167.00 \pm 16.08$ & $153.79 \pm 16.08$ & $155.09 \pm 16.08$ & $147.75 \pm 15.54$ & $145.93 \pm 15.54$ & $155.09 \pm 15.54$ & 0.71 \\
\hline Mean CD34+ve & $2527.64 \pm 508.69$ & $3128.79 \pm 508.69$ & $3157.36 \pm 508.69$ & $2452.07 \pm 491.44$ & $2031.40 \pm 491.44$ & $2682.93 \pm 491.44$ & 0.32 \\
\hline$\%$ of CD34+ve & $1.01 \pm 0.19$ & $1.04 \pm 0.17$ & $1.23 \pm 0.21$ & $0.94 \pm 0.19$ & $0.85 \pm 0.22$ & $0.93 \pm 0.17$ & 0.75 \\
\hline$\%$ of CD31+ve & $2.85 \pm 0.33$ & $2.47 \pm 0.44$ & $2.16 \pm 0.43$ & $2.75 \pm 0.33$ & $2.34 \pm 0.38$ & $2.58 \pm 0.35$ & 0.59 \\
\hline CD34+CD184 & $0.57 \pm 0.09$ & $0.45 \pm 0.10$ & $0.61 \pm 0.11$ & $0.43 \pm 0.09$ & $0.33 \pm 0.10$ & $0.37 \pm 0.09$ & 0.62 \\
\hline CFU & $10.30 \pm 2.03$ & $13.62 \pm 2.10$ & $13.61 \pm 2.19$ & $6.08 \pm 1.95$ & $7.62 \pm 1.95$ & $7.53 \pm 1.89$ & 0.82 \\
\hline SDF $10 \mathrm{NG}$ & $0.20 \pm 0.031$ & $0.20 \pm 0.031$ & $0.23 \pm 0.03$ & $0.15 \pm 0.03$ & $0.15 \pm 0.03$ & $0.26 \pm 0.03$ & 0.26 \\
\hline SOD2 & $0.97 \pm 0.23$ & $1.45 \pm 0.23$ & $0.92 \pm 0.24$ & $1.00 \pm 0.22$ & $1.05 \pm 0.24$ & $1.36 \pm 0.24$ & 0.22 \\
\hline Catalase & $-0.03 \pm 0.25$ & $-0.09 \pm 0.25$ & $-0.61 \pm 0.26$ & $-0.55 \pm 0.23$ & $-0.55 \pm 0.26$ & $0.20 \pm 0.26$ & 0.04 \\
\hline GPX35 & $2.00 \pm 0.54$ & $2.00 \pm 0.54$ & $1.71 \pm 0.54$ & $2.00 \pm 0.47$ & $1.64 \pm 0.50$ & $2.25 \pm 0.54$ & 0.68 \\
\hline CXCL12 & $1.00 \pm 0.16$ & $0.53 \pm 0.17$ & $0.33 \pm 0.17$ & $1.00 \pm 0.15$ & $0.60 \pm 0.16$ & $0.63 \pm 0.18$ & 0.59 \\
\hline CXCR4 & $1.06 \pm 0.17$ & $1.03 \pm 0.17$ & $0.90 \pm 0.18$ & $1.00 \pm 0.16$ & $1.03 \pm 0.18$ & $1.58 \pm 0.18$ & 0.06 \\
\hline EDN1 & $1.22 \pm 0.27$ & $1.05 \pm 0.29$ & $1.03 \pm 0.29$ & $1.00 \pm 0.24$ & $0.79 \pm 0.27$ & $2.10 \pm 0.27$ & 0.02 \\
\hline VEGEF-A & $0.95 \pm 0.17$ & $1.46 \pm 0.18$ & $0.83 \pm 0.19$ & $1.00 \pm 0.17$ & $0.86 \pm 0.18$ & $1.12 \pm 0.19$ & 0.04 \\
\hline PECAM & $0.94 \pm 0.11$ & $1.43 \pm 0.11$ & $0.89 \pm 0.12$ & $1.00 \pm 0.11$ & $0.87 \pm 0.12$ & $1.28 \pm 0.12$ & 0.002 \\
\hline KDR & $1.00 \pm 0.18$ & $0.53 \pm 0.18$ & $0.50 \pm 0.20$ & $1.00 \pm 0.18$ & $0.55 \pm 0.20$ & $1.16 \pm 0.20$ & 0.13 \\
\hline NOS3 & $1.11 \pm 0.21$ & $0.06 \pm 0.21$ & $-0.25 \pm 0.21$ & $0 \pm 0.18$ & $-0.23 \pm 0.19$ & $0.34 \pm 0.21$ & 0.08 \\
\hline Wilm's tumor & $6.52 \pm 0.65$ & $6.13 \pm 0.72$ & $6.36 \pm 0.75$ & $6.27 \pm 0.60$ & $6.68 \pm 0.62$ & $5.75 \pm 0.59$ & 0.45 \\
\hline Nephrin & $6.15 \pm 0.55$ & $5.10 \pm 0.62$ & $5.87 \pm 0.65$ & $5.39 \pm 0.51$ & $5.70 \pm 0.53$ & $5.24 \pm 0.50$ & 0.25 \\
\hline PODXL & $6.72 \pm 0.33$ & $6.08 \pm 0.38$ & $6.68 \pm 0.40$ & $7.05 \pm 0.31$ & $6.92 \pm 0.32$ & $6.62 \pm 0.30$ & 0.34 \\
\hline
\end{tabular}


serum based outcome measures are independent of body composition changes.

\section{Venous blood biochemistries}

Venous blood biochemistries were gathered, both through Labcorp of America and through serum ELISA and both standard of care and research labs were collected.

Detailed lab values of selected significant parameter are on Table 1 . We found statistically significant decrease in the Glucose levels in Canagliflozin group $(p=0.01)$, whereas an opposite effect is seen in placebo group. A sharp decrease in HbA1c levels from visit 1 to 2 in Canagliflozin group $(\mathrm{p}=0.0919)$ and overall decrease in HbA1c as compared to placebo group (Fig. 1) exert a beneficial effects on endothelial progenitor cell function. The inflammatory marker IL-6 levels in serum decreased significantly. As shown in the Fig. 1, in Canagliflozin treated subjects from visit 1 to visit 3 IL-6 levels reduced $(\mathrm{P}=0.05)$. Adiponectin and endocrine factor synthesized and released from adipose tissue. We have observed a significant increase in adiponectin levels $(\mathrm{p}=0.02)$ in Canagliflozin group, where as in placebo group adiponectin levels decreased from visit 1 to 3 (Fig. 1).

To study the effect of Canagliflozin on mitochondrial function serum NAD and NADH levels in placebo and Canagliflozin groups, we measured serum NAD and NADH levels from visit 3 . We did not see any significant difference between the groups (for Placebo $0.8454 \pm 0.3239$ and for Canagliflozin $0.7335 \pm 0.2730$ ). Serum ketone bodies, 3hydroxybutyric acid and acetoacetic acid were measured. As shown in the Table 1, there is no significant difference between the groups between either of the two ketone bodies.

\section{Arterial stiffness}

Stiffness of an artery significantly contributes to lack of pliability and contractility and is an important marker of increased peripheral resistance, diastolic dysfunction and systemic hypertension. It is associated with

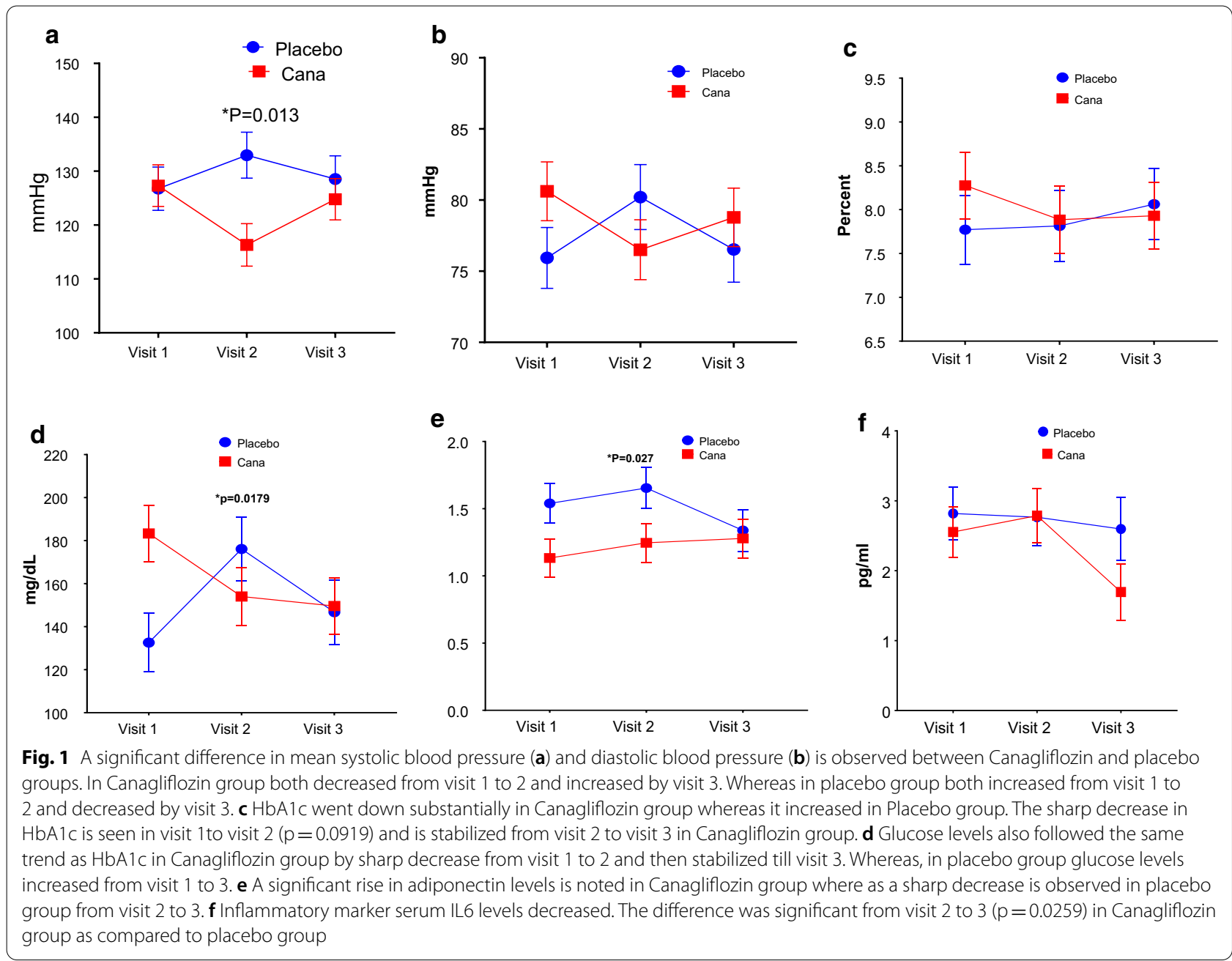


cardiovascular diseases in older individuals and is positively associated with hypertension, Coronary Artery Disease, stroke, heart failure and atrial fibrillation [41, 42]. Arterial stiffness is assessed using parameters such as AI adjusted for a heart rate of 75 (AI-75) and PWV. The mean PWV for canagliflozin group started at a lower level as compared to placebo group and is not significantly different from visit 1 to 3 . In the placebo group PWV is high at visit 1 and remains high until visit 3 (Table2).

Even though there is no significant difference in augmentation index AI [adjusted for a heart rate of 75 (AI75)] and PWV between placebo and Canagliflozin treated subjects, there was a significant reduction in mean systolic blood pressure $(\mathrm{p}=0.01)$, noted in Canagliflozin group as compared to Placebo group. Similarly significant decrease in mean diastolic blood pressure $(\mathrm{p}=0.02)$ was noted in Canagliflozin group as compared to placebo group.

\section{Adiposity}

Body composition measurement showed no statistically significant change amongst the group throughout the visits. As expected, given short duration of the treatment the subjects were asked to maintain activity level as advised by American Diabetic Association (ADA) for healthy living. There was no statistically significant change in hip to waist ratio, body weight and body fat percent amongst the treatment and control group.

\section{Cellular outcome measures}

\section{Characterization of endothelial progenitor cells (CD34+ve)}

To study the effect of SGLT2 inhibitor, Canagliflozin, on the endothelial progenitor cell number we counted the total CD34+ve cells by using Cellometer Mini (Nexcelom Biosciences, Lawrence, MA), after microbead column separation (BD Biosciences, San Jose, CA). As shown in Table 2, there is no statistical significance in difference in CD34+ve cell numbers between placebo and Canagliflozin group.

To discern the direct effect of Canagliflozin on CD34+ve cells percentage the values were adjusted for HbA1c. HbA1c adjusted value for CD34+ve cell number is statically significant $(p=0.0047)$. Consistent with this, HbA1C adjusted values for dual positive cells, CD34+ve/ CD184+ve cells, were also statistically significant $(p=0.0039)$ between the groups, with higher number in Canagliflozin group.

\section{CFU-Hill's colonies}

The CFU-Hill's colony formation was improved in the Canagliflozin treatment group. CFU numbers increased sharply from visit 1 to 2 and visit 1 to 3 (data not shown). HbA1c adjusted value for CFU is statically significant $(\mathrm{p}=0.042)$ with higher CFU count in canagliflozin group. In placebo group despite an increase in CFU number from visit 1 to 2 there was an overall decrease in CFU number from visit 1 to 3 .

\section{Migration response}

Next, we wanted to study the effect of Canagliflozin on migration of CD34+ve cells, using Bowden's chanber. The migratory response of CD34+ve cells to the chemotactic factor SDF1 $\alpha$ (concentration of $10 \mathrm{ng} / \mathrm{ml}$ ) increased in canagliflozin group as compared to placebo group. However from visit 2 to 3 there was a significant increase in migration of CD34+ve cells in canagliflozin group as compared to placebo group (Fig. 2).

\section{Gene expression analysis \\ The effect of Canagliflozin on the gene expression of CD34+ve cells}

We wanted to study the gene expression on CD34+ve cells for antioxidants SOD2 (superoxide dismutase 2), GPX3 (glutathione peroxidase 3), CAT (catalase) genes. The mean fold change in gene expression of all 3 antioxidants (Sod2, p $=0.22$, CAT, $\mathrm{p}=0.04, \mathrm{GPX} 3, \mathrm{p}=0.68$ ) that we studied were increased in canagliflozin group where as in placebo group it is decreased from visit 1 to visit 3 (Fig. 3a-c). The mean gene expression for endothelial markers VEGF-A $(\mathrm{p}=0.04), \operatorname{KDR}(\mathrm{p}=0.13)$ and PECAM1 $(\mathrm{p}=0.02)$ had increased significantly in Canagliflozin group from visit 1 to visit 3 (Fig. $4 \mathrm{a}-\mathrm{c}$ ) as compared to placebo group. Since the prominent endothelial gene expression (VEGF) is increased significantly, we were interested to see the expression of endothelial functional genes NOS3(endothelial nitric oxide synthase). Over all there is a trend in increase in NOS3 expression $(\mathrm{P}=0.08)$ in Canagliflozin treated group as compared to placebo group though no statistical significant difference was observed between the groups (Fig. 4d). We also checked the inflammatory markers and we did not see any differences in the inflammatory markers IL6 and TNF- $\alpha$ expression between the groups (data not shown), however the serum IL-6 levels decreased in Canagliflozin treated group as compared to placebo group (Fig. 1f). This is difference is statistically significant from visit 2 to visit $3(\mathrm{p}=0.0259)$.

\section{Urine podocyte function marker \\ Quantification of exosomal proteins in urine samples by western blot}

Our recent study using Linagliptin showed protection from renal injury with patients taking Linagliptin for a duration of 12 weeks [44]. However the difference between Linagliptin and placebo was not picked up by urine albumin: creatine ratio but was noted on assessing 

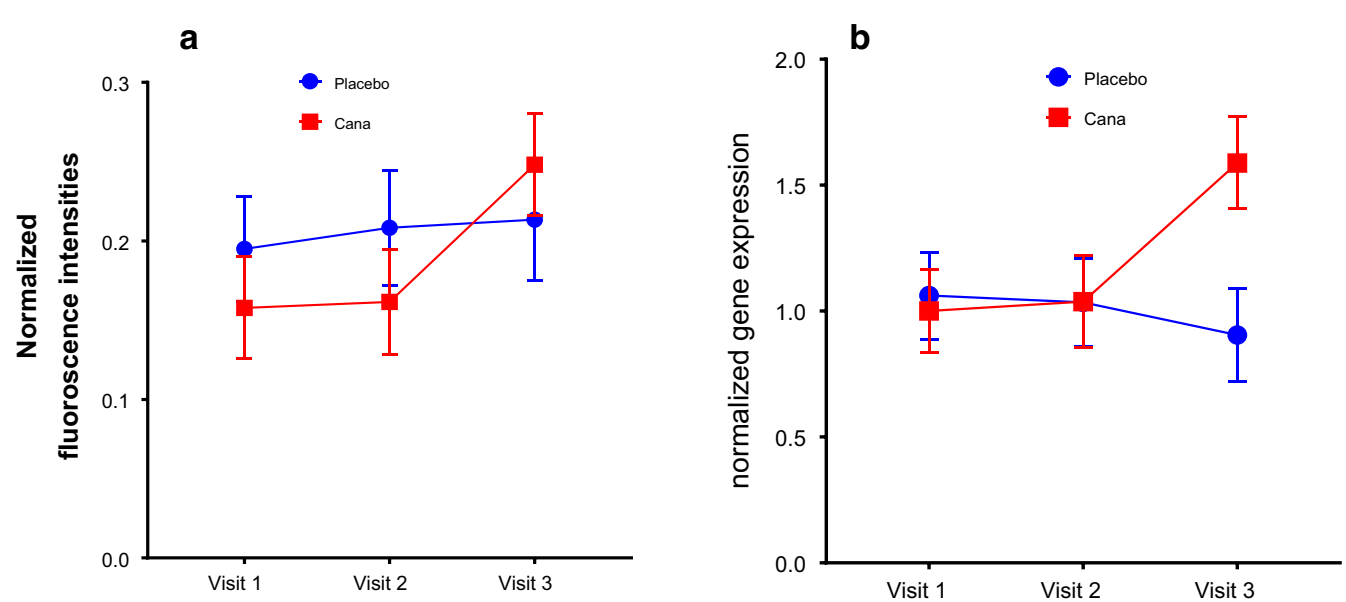

Fig. 2 Migration Assay in response to SDF1alpha at $10 \mathrm{ng} / \mathrm{ml}$ concentration and gene expression of SDF1a receptor, CXCR-4 on CD34+ve cells. a Mean migration of CD34+ve cells towards SDF1-a (at $10 \mathrm{ng} / \mathrm{ml}$ ) increased from visit 1 to 3 in Canagliflozin group as compared to placebo group (for visit 1 to $3 p=0.03$ and for visit $2-3 p=0.05)$. $\mathbf{b}$ CXCR4 gene expression on CD34 positive cells is increased ( $p=0.06)$ in Canagliflozin group as compared to placebo group at visit 3
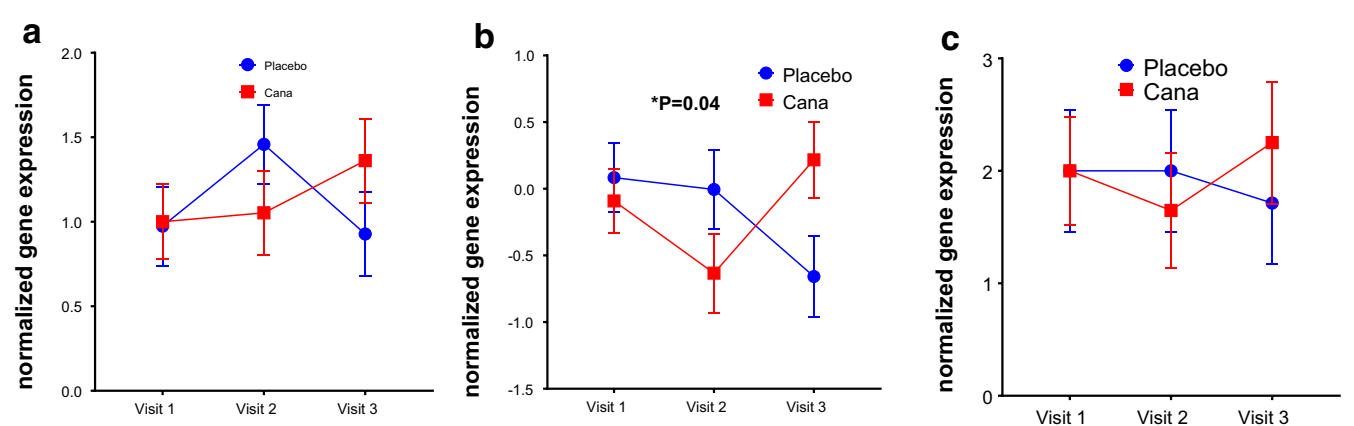

Fig. 3 The effect of Canagliflozin on CD34+ve cell antioxidants gene expression. Fold change in gene expression of antioxidants SOD2 (a) in CD34+ve cells are increased in Canagliflozin group from visit 1 to 3 . However, the same for the placebo group is decreased. Whereas the antioxidants, $\mathbf{b}$ Catalase (CAT) and c GPX3 gene expression increased in CD34+ve cells in Canagliflozin group from visit 2 to 3 . However, the same for the placebo group is decreased. Overall, all the antioxidant genes showed increased expression going from visit 2 to visit 3 , whereas the placebo there was a downward trend with catalase expression reaching statistical difference, overall

urinary exosome podocyte markers. We were interested to study the effect of Canagliflozin on podocyte health by quantifying the exosome expression for Nephrin, Wilm's Tumor (WT-1) and podocalaxyn like protein 1 (PODXL) in urine samples from placebo and Canagliflozin group. These three proteins are specific for podocyte health.

As described in the methods after isolating exosomes from urine samples of both placebo and canagliflozin group exosomes were identified as CD9 positive. As shown in the Fig. 5, the mean band intensities for the podocyte specific exosomal protein leak of PODXL and Nephrin and WT-1 were decreased from visit 1 to 3 in Canagliflozin group as compared to placebo group. Even though the difference in band intensities between the groups is not significant (taking all visits together) a trend in decrease intensities is observed in Canagliflozin treated group, particularly in visit 2 to visit 3 , indicating a late onset protection effect of Canagliflozin. Interestingly, the HbA1c adjusted value for Nephrin in Canagliflozin treated group was statically significant $(p=0.0133)$.

\section{Discussion \\ Primary outcome: cellular}

Reduction in CD34+ve cells is a consistent observation in people with $\mathrm{T} 2 \mathrm{D}$ and this defect may contribute to the development of chronic micro and macrovascular complications. Previous studies demonstrated that SGLT2 inhibitors reduce the risk of cardiovascular 

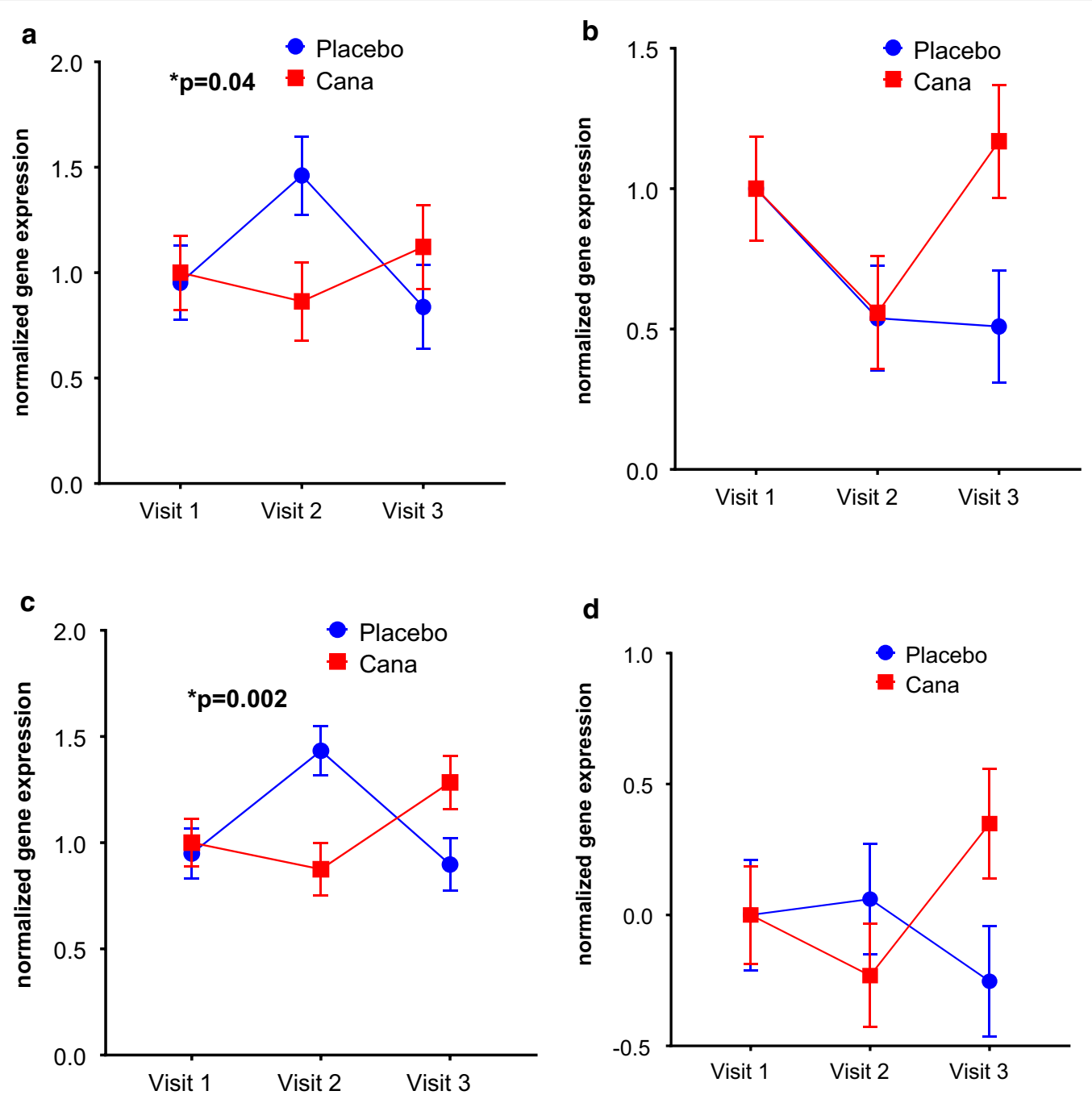

Fig. 4 The effect of Canagliflozin on CD34+ve cell Endothelial markers gene expression. Fold change in gene expression of Endothelial markers a VEGF-A, $\mathbf{b}$ KDR and $\mathbf{c}$ PECAM and endothelial function marker $\mathbf{d}$ NOS3 on CD34+ve cells increased significantly in Canagliflozin group from visit 1 to 3. However, the same for the placebo group is decreased. Gene expression is normalized to $18 \mathrm{~S}$
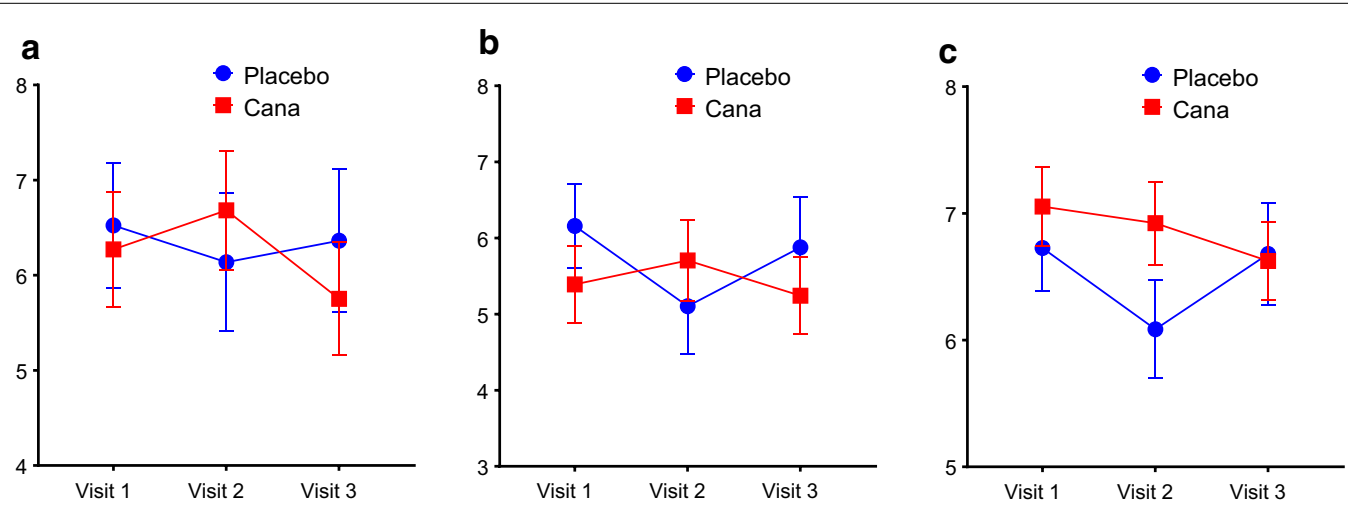

Fig. 5 Urinary exosome markers a Wilm's tumor-1 (WT-1), b Nephrin and c Podocalyxin (PODXL) were identified by Western blot and followed by quantification. A trend in reduced mean band intensities for Urinary exosome markers are observed in Canagliflozin group from visit 1 to visit 3 as compared to placebo group 
events in patients with T2D and high cardiovascular risk $[45,46]$. Despite several attempts to explain such striking cardiovascular protection exerted by SGLT2 inhibitors we still have a poor understanding of the potential mechanisms at work. Therefore, in this study we attempted to test whether SGLT2 inhibitor Canagliflozin exert beneficial effects on endothelial progenitor cells which are considered as biomarkers and have a role in the pathogenesis of chronic diabetic complications. We investigated the effect of SGLT2 inhibitor Canagliflozin in addition to metformin and/or Insulin on CD34+ve EPCs and CD34+ve CD184+ve cells as a marker for vascular endothelial function. Our data shows canagliflozin did not increase the endothelial progenitor cells in this short time 16 week study. CD34+ve cells decreased from visit 1 to 2 and then increased to slightly higher by the end of Visit 3 in canagliflozin group. The decrease in EPCs is in parallel with the recent study on effect of SGLT2 inhibitors on EPCs [36]. Even though, CD34+ve cells are not increased, in number, statistically, the expression of CXCR4 and SDF1 (CD184+ve) expression are increased in the CD34+ve cells. This finding is correlated with the increased migratory response to SDF1- $\alpha$ of CD34+ve cells in canagliflozin group (Fig. 2b). It is likely that long term treatment with canagliflozin will increase the function of CD34+ve cells along with good glycemic control [46].

Next, in order to understand the effect of Canagliflozin on gene expression of CD34+ve cells we performed qPCR analysis. We looked at pathways involving inflammation, oxidative stress, EPC chemotaxis pathways and endothelial function markers, in CD34+ve cells obtained post magnetic column separation of MNCs. We found the expression of SDF1- $\alpha$ receptor CXCR4 expression is very close to statistical significance in Canagliflozin treatment. This was an unexpected result as we did not expect an SGLT2i to have effect of stem cell migration, unlike DPP4i treatment where migration can be expected to increase secondary to increased SDF1a increased bioavailability. Antioxidants and endothelial markers are upregulated in Canagliflozin treatment group, indicating improved vascular health. The upregulation of endothelial markers, VEGF-A and its receptor KDR indicates VEGF signaling most likely plays an important role in vasculogenesis and tissue repair upon Canagliflozin treatment. Interestingly eNOS expression increased significantly from visit 2 to visit 3 in Canagliflozin group. Another interesting findings were increase in serum adiponectin levels by ELISA and reduction in serum IL-6 values particularly between visit 2 to visit 3 . These two results concurrently indicate improved endothelial health following canagliflozin treatment (Fig. 1e, f).
As nephropathy is an important progressive complication of T2DM we specifically looked at markers beyond urine microalbumin: creatinine ratio which did not show a difference between the groups. However, when we examined a more sensitive marker such as urine exosomes that specifically look at podocyte derived protein such as podocalcyxin, nephrin and Wilm's tumor we noted that canagliflozin appears to reduce these podocyte proteins particularly between visit 2 to 3 .

Lastly, we wanted to examine any possible differences in ketone bodies between placebo and low dose canagliflozin as the latter has been shown to cause euglycemic ketosis [47], however at this dose we did not note any difference in serum ketone body levels. There are reports of canagliflozin influencing mitochondrial function via AMPK-Sirt1-Pgc-1 $\alpha$ signalling pathway [48] however we will note any differences in NAD: NADH ratio, however it will be worthwhile to investigate into these effects at higher doses.

\section{Limitations of our study}

Limitations of our pilot study may include the relatively short duration of 16-week Canagliflozin therapy, which may have been inadequate to see significant changes in certain clinical and cellular parameters. This may have been also because of the small sample size. However, we were satisfied to note statistical significance in various cellular outcomes and even in some serum based markers. Further studies with a larger population and longer duration may be helpful to further define the mechanisms behind our findings.

\section{Conclusion}

Canagliflozin when added to subjects with Type 2 Diabetes along with metformin and/or Insulin, demonstrates a functional improvement of CD34+ Endothelial Progenitor Cell migratory function through increased CD34/ CXCR4 positivity. Canagliflozin also promotes improvement in blood pressure, arterial stiffness and serum biochemical markers that indicates improved endothelial function.

We believe CD34+ cells can act as a valuable biomarker for assessment of endothelial function, in a setting of diabetes and can help provide valuable clinical information leading to appropriate therapeutic intervention choices. It may also allow an individualistic approach to diabetes medication choice rather than standard serum based assays with set normal ranges.

\section{Abbreviations}

Al: Augmentation Index; Al-75: Augmentation Index adjusted for a heart rate of 75; AP: Augmentation Pressure; AS: Arterial stiffness; BCl-2: B-cell lymphoma 2; BMI: Body mass index; BUN: Blood urea nitrogen; CAT: Catalase; CDKN1A: 
Cyclin-dependent kinase inhibitor 1A; CD34: Progenitor marker; CFU: Colony forming units; CVD: Cardiovascular disease; CXCR4: C-X-C Motif Chemokine Receptor 4; DBP: Diastolic blood pressure; DPP-4 inhibitors: Dipeptidyl peptidase-4 inhibitors; EDN-1: Endothelin 1; eGFR: Estimated glomerular filtration rate; ELISA: Enzyme-Linked Immunosorbent Assay; eNOS: Nitric oxide synthase 3 (endothelial cell); EPCs: Endothelial progenitor cells; FFM: Fat free mass; GAPDH: Glyceraldehyde-3-phosphate dehydrogenase; GLP1: Glucagon like peptide 1; GPX1: Glutathione peroxidase 1; HbA1C: Hemoglobin A1C, glycated hemoglobin test; IGF1: Insulin-like growth factor 1; IL-6: Interleukin 6; kg: Kilograms (weight); Ibs: Pounds (weight); MNCs: Mononuclear cells; PECAM1: Platelet and endothelial cell adhesion molecule 1; PWA: Pulse wave analysis; PWV: Pulse wave velocity; qRT-PCR: Quantitative reverse transcriptase polymerase chain reaction; REE: Resting energy expenditure; SBP: Systolic blood pressure; SDF1a: Stromal cell-derived factor-1 a; SOD1, SOD2: Super oxide dismutase 1 \& 2; TBW:Total body water; TNFa:Tumor Necrosis Factor a; TP53: Tumor protein p53; VEGF: Vascular endothelial growth factor; VEGFA: Vascular endothelial growth factor A; VEGFR2: Vascular endothelial growth factor receptor 2; VO2: Maximal oxygen consumption; 18S: 18S Ribosomal RNA.

\section{Acknowledgements}

We would like to thank Ms. Magan Fasso and Ms. Bethany Batistich in helping with recruitment of subjects. We would also like to thank Drs. Jill Paulson and Rohit Jain of the Endocrinology division at GW in helping to identify suitable subjects with type 2 diabetes and Dr. Eric Nylen of Washington DC Veteran's Affairs Medical Center for proof-reading the manuscript. The author(s) meet criteria for authorship as recommended by the International Committee of Medical Journal Editors (ICMJE). This study was supported by Janssen Pharmaceuticals, Inc. investigator initiated grant to Dr. S Sen. Janssen had no role in the design, analysis or interpretation of the results in this study; Janssen was given the opportunity to review the manuscript for medical and scientific accuracy as it relates to Janssen substances, as well as intellectual property considerations.

\section{Authors' contributions}

SRN performed the experiments and were responsible for collection and compilation of data, analysis of data, and in writing the manuscript. NK contributed to cellular outcome measures, compilation of the data and helped write the manuscript. HA responsible for collection of clinical data and contributed to clinical assessments. BB conducted the urine exosome-based assay, including related data compilation and analysis. MF has contributed in collection and complication of the clinical and cellular data sets. NA and AE contributed in performing cellular experiments particularly the gene expression studies. RA performed the statistical analyses of the data set, and SS designed and supervised the study and revised the manuscript. All authors read and approved the final manuscript

\section{Funding}

Janssen Scientific Affairs, LLC.

\section{Availability of data and materials}

All associated data will be available to the public, as requested. Demography of subjects including detailed description of baseline characteristics and parameters have been included in the Appendices.

\section{Ethics approval and consent to participate}

Our study was approved by the George Washington University Institutional Review Board and was conducted in accordance with Good Clinical Practices of the National Institutes of Health. Signed informed consent was obtained by all subjects prior to engaging in any research.

\section{Consent for publications}

Not applicable.

\section{Competing interests}

The authors declare that they have no competing interests.

\section{Author details}

${ }^{1}$ The GW Medical Faculty Associates, Washington, DC, USA. ${ }^{2}$ Department of Medicine, The George Washington University, 2300 Eye Street, SMHS, Room 462, Washington, DC 20037, USA. ${ }^{3}$ Department of Medicine and Endocrinology, Veterans Affairs Medical Center, Washington, DC, USA.

\section{Appendix}

\section{Inclusion criteria}

1. Adults aged $30-70$ years.

2. Diagnosis of type 2 diabetes within the previous 15 years using criteria of the American Diabetes Association.

3. Currently treated with a stable dose of Insulin, Metformin (1-2 g/day), or a stable combination of the two as therapy.

4. HbA1C between 7 and $10 \%$ (both inclusive).

5. BMI 25 to $39.9 \mathrm{~kg} / \mathrm{m}^{2}$ (both inclusive).

\section{Exclusion criteria}

1. Implanted devices (e.g., pacemakers) that may interact with Tanita scale.

2. Previous coronary or cerebrovascular event within 6 months of screening or active or clinically significant coronary and/or peripheral vascular disease.

3. Low hematocrit ( $<28$ units).

4. Pre-existing liver disease and/or ALT and AST > 2.5X's UNL.

5. CKD stage 4 and,

6. History of pancreatitis, or cancer (except basal cell carcinoma and cancer that is cured or not active or being treated in the past 5 years).

7. Statin use started or dose change in the last 3 months.

8. Use of oral anti-diabetic medication other than Metformin, or Insulin.

9. Use of consistent long-term steroid medication in the last 3 months (oral, inhaled, injected).

10. Systolic BP $>140 \mathrm{mmHg}$ and Diastolic $\mathrm{BP}>90 \mathrm{mmHg}$.

11. Active wounds or recent surgery within 3 months.

12. Inflammatory disease, or chronic current use of anti-inflammatory drugs within the last 3 months.

13. Triglycerides $>450 \mathrm{mg} / \mathrm{dl}$.

14. Untreated hyper/hypothyroidism.

15. Auto antibody confirmed type 1 diabetes.

Additionally, patients who are active smokers, patients who are pregnant, nursing women, and post-menopausal women who are on hormone replacement therapy will be excluded.

Patients on low dose oral contraceptives will be allowed to participate as these formulations contain lesser amount of estrogens. 
Received: 12 November 2020 Accepted: 1 February 2021

Published online: 13 February 2021

\section{References}

1. IDF Diabetes Atlas. 9th edition; 2019. https://www.diabetesatlas.org/en/ Accessed 09 Nov 2020.

2. US Renal Data System Annual Data Report; 2019. https://www.usrds.org/ annual-data-report/. Accessed 09 Nov 2020.

3. Amorim RG, et al. Kidney disease in diabetes mellitus: cross-linking between hyperglycemia, redox imbalance and inflammation. Arq Bras Cardiol. 2019;112:5.

4. Giacco F, Brownlee M. Oxidative stress and diabetic complications. Circ Res. 2010;107:9.

5. Meza CA, et al. Endothelial dysfunction: is there a hyperglycemia-induced imbalance of NOX and NOS? Int J Mol Sci. 2019;20:15

6. Perlman RL, Fredri F, Liu L, et al. Quality of life in chronic kidney disease (CKD): a cross-sectional analysis in the Renal Research Institute-CKD study. Am J Kidney Dis. 2005;45:4

7. Rosenthal $\mathrm{N}$, et al. Canagliflozin: a sodium glucose co-transporter 2 inhibitor for the treatment of type 2 diabetes mellitus. Ann N Y Acad Sci. 2015;1358:28-43.

8. van Bommel EJ, et al. SGLT2 inhibition in the diabetic kidney-from mechanisms to clinical outcome. Clin J Am Soc Nephrol. 2017;12:4.

9. Huri HZ, Lim LP, Lim SK. Glycemic control and antidiabetic drugs in type 2 diabetes mellitus patients with renal complications. Drug Des Devel Ther. 2015;9:4355.

10. Perkovic $V$, et al. Renal effects of canagliflozin in type 2 diabetes mellitus. Curr Med Res Opin. 2015;31:12.

11. Abdul-Ghani MA, DeFronzo RA. Inhibition of renal glucose reabsorption: a novel strategy for achieving glucose control in type 2 diabetes mellitus. Endocr Pract. 2008;14:6.

12. Lo MC, Lansang MC. Recent and emerging therapeutic medications in type 2 diabetes mellitus: incretin-based, pramlintide, colesevelam, SGLT2 inhibitors, tagatose, succinobucol. Am J Ther. 2013;20:6.

13. Naznin F, et al. Canagliflozin, a sodium glucose cotransporter 2 inhibitor, attenuates obesity-induced inflammation in the nodose ganglion, hypothalamus, and skeletal muscle of mice. Eur J Pharmacol. 2017:794:37-44.

14. Rossing P, de Zeeuw D. Need for better diabetes treatment for improved renal outcome. Kidney Int Suppl. 2011;120:S28-32.

15. Vallon V, Sharma K. Sodium-glucose transport: role in diabetes mellitus and potential clinical implications. Curr Opin Nephrol Hypertens. 2010;19:5.

16. Ryan PB, et al. Comparative effectiveness of canagliflozin, SGLT2 inhibitors and non-SGLT2 inhibitors on the risk of hospitalization for heart failure and amputation in patients with type 2 diabetes mellitus: a real-world meta-analysis of 4 observational databases (OBSERVE-4D). Diabetes Obes Metab. 2018;20:1

17. Ali BH, et al. Effects of the SGLT-2 inhibitor canagliflozin on adenineinduced chronic kidney disease in rats. Cell Physiol Biochem. 2019;52:1.

18. Mahaffey KW, et al. Canagliflozin for primary and secondary prevention of cardiovascular events: results from the CANVAS program (canagliflozin cardiovascular assessment study). Circulation. 2018;137:4.

19. Neal B, et al. Canagliflozin and cardiovascular and renal events in type 2 diabetes. N Engl J Med. 2017;377:7

20. Neuen B, et al. Cardiovascular and renal outcomes with canagliflozin according to baseline kidney function. Circulation. 2018;138:15.

21. Patorno E, et al. Cardiovascular outcomes associated with canagliflozin versus other non-gliflozin antidiabetic drugs: population based cohort study. BMJ. 2018:360:k119.

22. Rådholm K, et al. Canagliflozin and heart failure in type 2 diabetes mellitus: results from the CANVAS program. Circulation. 2018;138:5.

23. Skelley JW, Carter BS, Roberts MZ. Clinical potential of canagliflozin in cardiovascular risk reduction in patients with type 2 diabetes. Vasc Health Risk Manag. 2018;14:419.

24. Mancini SJ, et al. Canagliflozin inhibits interleukin-1 $\beta$-stimulated cytokine and chemokine secretion in vascular endothelial cells by AMP-activated protein kinase-dependent and -independent mechanisms. Sci Rep. 2018;8:1.

25. Sayour AA, et al. Acute canagliflozin treatment protects against in vivo myocardial ischemia-reperfusion injury in non-diabetic male rats and enhances endothelium-dependent vasorelaxation. J Transl Med. 2019;17:1.

26. Sen S. Adult stem cells: beyond regenerative tool, more as a bio-marker in obesity and diabetes. Diabetes Metab J. 2019;43:6.

27. Gendron N, Smadja DM. Circulating endothelial cells: a new biomarker of endothelial dysfunction in hematological diseases. Ann Biol Clin. 2016;74:4.

28. Jain $\mathrm{R}$, Awal $H$, Sen $\mathrm{S}$. Using adult stem cells to monitor endothelial dysfunction in diabetes mellitus. J Diabetes Complicat. 2020;34(7):107588.

29. Sen S, Witkowski S, Lagoy A, Islam A. A six-week home exercise program improves endothelial function and CD34+ circulating progenitor cells in patients with pre-diabetes. J Endocrinol Metab. 2015:5:1-2.

30. Rigato M, Fadini GP. Circulating stem/progenitor cells as prognostic biomarkers in macro- and microvascular disease: a narrative review of prospective observational studies. Curr Med Chem. 2018;25:35.

31. Rohban R, Prietl B, Pieber TR. Crosstalk between stem and progenitor cellular mediators with special emphasis on vasculogenesis. Transfus Med Hemother. 2017:44:3.

32. Prokoph S, et al. Sustained delivery of SDF-1 a from heparin-based hydrogels to attract circulating pro-angiogenic cells. Biomaterials. 2012;33:19.

33. Shen $L$, et al. A novel mechanism for endothelial progenitor cells homing: the SDF-1/CXCR4-Rac pathway may regulate endothelial progenitor cells homing through cellular polarization. Med Hypotheses. 2011:76:2.

34. Kutikhin AG, et al. Shear stress: an essential driver of endothelial progenitor cells. J Mol Cell Cardiol. 2018;118:46-69.

35. Medina RJ, et al. Endothelial progenitors: a consensus statement on nomenclature. Stem Cells Transl Med. 2017:6:5

36. Bonora BM, et al. Effects of SGLT2 inhibitors on circulating stem and progenitor cells in patients with type 2 diabetes. J Clin Endocrinol Metab. 2018;103:10.

37. Mudaliar S, Alloju S, Henry RR. Can a shift in fuel energetics explain the beneficial cardiorenal outcomes in the EMPA-REG OUTCOME study? A unifying hypothesis. Diabetes Care. 2016;39:7.

38. Park SH, et al. Empagliflozin improved systolic blood pressure, endothelia dysfunction and heart remodeling in the metabolic syndrome ZSF1 rat. Cardiovasc Diabetol. 2020;19:1.

39. Steven $S$, et al. The SGLT2 inhibitor empagliflozin improves the primary diabetic complications in ZDF rats. Redox Biol. 2017:13:370-85.

40. Uthman L, et al. Empagliflozin and dapagliflozin reduce ROS generation and restore NO bioavailability in tumor necrosis factor a-stimulated human coronary arterial endothelial cells. Cell Physiol Biochem. 2019;53:5.

41. Stoner L, Young JM, Frye S. Assessments of arterial stiffness and endothelial function using pulse wave analysis. Int J Vasc Med. 2012;2012:903107. https://doi.org/10.1155/2012/903107.

42. Khiyami AM, Dore FJ, Mammadova A, Amdur RL, Sen S. The correlation of arterial stiffness with biophysical parameters and blood biochemistry. Metab Syndr Relat Disord. 2017;15(4):178-82.

43. Dore FJ, Domingues CC, Ahmadi N, Kundu N, Kropotova Y, Houston S, Rouphael C, Mammadova A, Witkin L, Khiyami A, Amdur RL, Sen S. The synergistic effects of saxagliptin and metformin on CD34+ endothelial progenitor cells in early type 2 diabetes patients: a randomized clinical trial. Cardiovasc Diabetol. 2018;17(1):65.

44. Awal HB, Nandula SR, Domingues CC, Dore FJ, Kundu N, Brichacek B, Fakhri M, Elzarki A, Ahmadi N, Safai S, Fosso M, Amdur RL, Sen S. Linagliptin, when compared to placebo, improves CD34+ve endothelial progenitor cells in type 2 diabetes subjects with chronic kidney disease taking metformin and/or insulin: a randomized controlled trial. Cardiovasc Diabetol. 2020;19:1

45. Fadini GP, Avogar A. SGLT2 inhibitors and amputations in the US FDA adverse event reporting system. Lancet Diabetes Endocrinol. 2017;5:9.

46. Fadini GP et al. Optimized glycaemic control achieved with add-on basal insulin therapy improves indexes of endothelial damage and regeneration in type 2 diabetic patients with macroangiopathy: a randomized crossover trial comparing detemir versus glargine. Diabetes Obes Metab. 2011;13:8. 
47. Thiruvenkatarajan V, Meyer EJ, Nanjappa N, Van Wijk RM, Jesudason D. Perioperative diabetic ketoacidosis associated with sodium-glucose cotransporter-2 inhibitors: a systematic review. Br J Anaesth. 2019;123:1.

48. Yang X, Liu Q, Li Y, Tang Q, Wu T, Chen L, Pu S, Zhao Y, Zhang G, Huang C, Zhang J, Zhang Z, Huang Y, Zou M, Shi X, Jiang W, Wang R, He J. The diabetes medication canagliflozin promotes mitochondrial remodelling of adipocyte via the AMPK-Sirt1-Pgc-1 a signalling pathway. Adipocyte. 2020;9:1.

\section{Publisher's Note}

Springer Nature remains neutral with regard to jurisdictional claims in published maps and institutional affiliations.
Ready to submit your research? Choose BMC and benefit from:

- fast, convenient online submission

- thorough peer review by experienced researchers in your field

- rapid publication on acceptance

- support for research data, including large and complex data types

- gold Open Access which fosters wider collaboration and increased citations

- maximum visibility for your research: over $100 \mathrm{M}$ website views per year

At BMC, research is always in progress.

Learn more biomedcentral.com/submissions 